\title{
Hiperendemicidad de fasciolosis y factores de riesgo en niños de edad escolar del distrito de Orurillo, Puno
}

\author{
Hyperendemicity of fascioliasis and risk factors in school-age children from the \\ Orurillo district, Puno
}

\author{
William Quispe P. ${ }^{1}$, María Beltrán F. ${ }^{1}$, Nury Vargas M. ${ }^{1}$, José Cabanillas A. ${ }^{2}$, \\ Elizabeth Sánchez R. ${ }^{1}$, Aldo Valderrama P.,4
}

\section{Resumen}

\begin{abstract}
El objetivo del estudio fue determinar la asociación de fasciolosis en niños de edad escolar con parásitos intestinales, consumo de alimentos y crianza de animales. La investigación fue de tipo analítico, prospectivo, transversal y de nivel relacional. Se obtuvieron 295 muestras serológicas y coprológicas y se realizó una entrevista epidemiológica a niños de ambos sexos, de 3 a 19 años de 23 instituciones de educación inicial, primaria y secundaria, con el consentimiento informado de sus progenitores. El diagnóstico serológico se realizó mediante la técnica de inmunoblot IgG y la prueba de ELISA IgG y el diagnóstico coprológico se realizó con la técnica de sedimentación rápida modificada por Lumbreras. Los factores de riesgo se obtuvieron mediante entrevista epidemiológica. La prevalencia de fasciolosis fue de $11.2 \%\left(\mathrm{IC}_{95 \%}=7.4-14.9 ; 33 / 295\right)$. Se encontró diferencias significativas entre instituciones educativas y comunidades de procedencia. Tanto en análisis univariado como el multivariado determinaron que beber agua de pozo y criar cuyes estuvieron asociados a fasciolosis.
\end{abstract}

Palabras clave: fasciolosis, niños, parásitos intestinales, crianza de animales, consumo de alimentos

${ }^{1}$ Instituto Nacional de Salud, Lima, Perú

${ }^{2}$ Universidad Peruana Cayetano Heredia, Lima, Perú

${ }^{3}$ Universidad Nacional Micaela Bastidas de Apurímac, Abancay, Perú

${ }^{4}$ E-mail: avalderrama@unamba.edu.pe

Recibido: 21 de enero de 2021

Aceptado para publicación: 10 de agosto de 2021

Publicado: 27 de octubre de 2021

CLos autores. Este artículo es publicado por la Rev Inv Vet Perú de la Facultad de Medicina Veterinaria, Universidad Nacional Mayor de San Marcos. Este es un artículo de acceso abierto, distribuido bajo los términos de la licencia Creative Commons Atribución 4.0 Internacional (CC BY 4.0) [https:// creativecommons.org/licenses/by/4.0/deed.es] que permite el uso, distribución y reproducción en cualquier medio, siempre que la obra original sea debidamente citada de su fuente original 
The aim of this study was to determine the association of fasciolosis in school-age children with intestinal parasites, food consumption and animal husbandry. The research was analytical, prospective, cross-sectional, and relational. In total, 295 serological and stool samples were obtained, and an epidemiological interview was carried out on children of both sexes, aged 3 to 19 years from 23 institutions of initial, primary and secondary education, with the informed consent of their parents. The serological diagnosis was made using the IgG immunoblot technique and the IgG ELISA test and the stool diagnosis was made with the rapid sedimentation technique modified by Lumbreras. Risk factors were obtained by epidemiological interview. The prevalence of fasciolosis was $11.2 \%$ (95\% CI $=7.4-14.9 ; 33 / 295)$. Significant differences were found between educational institutions and communities of origin. Both in univariate and multivariate analyses determined that drinking well water and raising guinea pigs were associated with fasciolosis.

Key words: fascioliasis, children, intestinal parasites, animal husbandry, food consumption

\section{INTRODUCCIÓN}

La fasciolosis es ocasionada por el trematodo hermafrodita Fasciola hepatica (Acha y Szyfres, 2003) y afecta a una gran variedad de especies animales incluyendo al hombre (Natividad y Terashima, 2008). Esta parasitosis tiene una distribución mundial, $\mathrm{y}$ se considera que entre 2.4 y 17 millones de personas estarían afectados y 91 millones estarían en riesgo de infectarse, mayormente en Perú, Bolivia y Ecuador (Marcos et al., 2005). La infección se obtiene especialmente al comer berros $u$ otros vegetales o tomar agua contaminada con metacercarias (Espinoza et al., 2010), por lo que se le considera un problema grave para la salud pública, así como una enfermedad desatendida.

En el Perú, esta enfermedad se ha ido incrementando en forma sustancial desde la década del 80, habiendo sido advertida como una enfermedad de transmisión vectorial con la más extensa presentación (Marcos, 2007). Las prevalencias más elevadas, tanto en personas como en animales se observa en los valles interandinos hasta los $4500 \mathrm{msnm}$, es- pecialmente en las regiones de Puno, Cajamarca, Cuzco, La Libertad, Arequipa, Apurímac, Huánuco, Piura, Lambayeque, Ayacucho y Junín (Jiménez et al., 2011). La transmisión se centra en la población rural dedicada a la agricultura, siendo los niños en edad escolar los más perjudicados (Martínez et al., 2006). El parásito requiere de un reservorio y un huésped intermediario para completar su ciclo biológico (Jiménez et al., 2011). El conocimiento de la epidemiología de esta parasitosis en el distrito de Orurillo, Puno, es escaso; sin embargo, la zona exhibe características sociales y ambientales conformes a zonas hiperendémicas del Perú (Valderrama, 2016; Merino y Valderrama, 2017). En consecuencia, el objetivo del estudio fue determinar la prevalencia de fasciolosis en niños y su asociación con parásitos intestinales, consumo de alimentos y crianza de animales en la zona.

\section{Materiales y Métodos}

Orurillo es uno de los nueve distritos de la provincia de Melgar, situada en la región Puno, en el Sudeste de Perú y a una altitud 
media de $3890 \mathrm{~m}$. Tiene una superficie de $379.05 \mathrm{~km}^{2}$, una población de 7651 habitantes y solo 721 domicilios disponen de agua potable en red pública (INEI, 2020). La temperatura diaria promedio máxima es de 16 ${ }^{\circ} \mathrm{C}$ y la mínima es de $0{ }^{\circ} \mathrm{C}$ (SENAMHI, 2020).

Los análisis coprológicos y serológicos se realizaron en el Laboratorio de Zoonosis Parasitaria del Instituto Nacional de Salud (INS), Lima, entre agosto de 2013 y febrero de 2014. El tamaño mínimo de muestra fue estimado en 265 niños de 3 a 19 años. Se realizó un muestreo simple aleatorio, distribuido proporcionalmente entre las 23 instituciones educativas de educación inicial, primaria y secundaria del distrito, conforme al censo escolar del Ministerio de Educación (MINEDU, 2017); sin embargo, se llegaron a tomar 295 muestras serológicas y coprológicas

La toma de las muestras se consiguió en coordinación con la Dirección Regional de Educación y los directores de las instituciones educativas. La selección de los niños se hizo en forma aleatoria. Se les entregó un kit conteniendo el frasco para depositar la muestra de heces, la ficha de asentimiento del niño y la ficha de consentimiento informado del padre o apoderado. Así mismo, se realizaron actividades de sensibilización dirigidas a los padres de los niños, donde se les alcanzó material informativo impreso (tríptico) relacionado a la fasciolosis.

El diagnóstico serológico se realizó mediante una prueba de ELISA IgG y la técnica de inmunoblot IgG (IB). La técnica de ELISA-IgG fue utilizada como prueba de tamizaje empleando kits in house de ELISAIgG (Cornejo et al., 2010; Sánchez et al., 2010), utilizando el antígeno metabólico «crudo» de $F$. hepatica excretado/secretado (AMESFH) con una concentración proteica de $1 \mathrm{mg} / \mathrm{ml}$. La lectura se hizo con el lector de ELISA Biotek ELX800, aceptando a las muestras con absorbancias por encima del valor de corte como reactivas y a las muestras con absorbancias iguales o menores al valor de corte como no reactivas. Consecuentemente, los valores se hallaron con la cifra promedio de las densidades ópticas de cada placa, a las que se le sumó el valor de dos desviaciones estándar. Por otro lado, la técnica de inmunoblot (IB-IgG) se usó como prueba confirmatoria para el diagnóstico de fasciolosis (Sánchez et al., 2010), usando el antígeno metabólico total de $F$. hepatica excretado/secretado (AMESFH) a una concentración de $2.07 \mu \mathrm{g} / \mu \mathrm{l}$. Para esto, se diagnosticó como positiva a la muestra que presentó uno o más péptidos antígénicos de $\mathrm{Mr}$ entre 17-23 KDa (Escalante et al., 2011), la cual alcanza una sensibilidad de 97\% (IC95\%: 89.6-100) y una especificidad de $96.6 \%$ (IC95\%: 94.2-98.9) (Escalante et al., 2011).

El diagnóstico coprológico de $F$. hepatica y enteroparásitos se realizó utilizando la técnica de Sedimentación Rápida Modificada por Lumbreras (TSR). Este método de concentración por sedimentación sin centrifugación se basa en la gravidez de los huevos, los que precipitan a causa de su peso y tamaño (Maco et al., 2002; Beltrán et al., 2003). Por otro lado, los factores de riesgo se obtuvieron realizando una anamnesis mediante entrevista epidemiológica.

Para la sistematización y análisis de la información se utilizó Excel 2010 y el programa SPSS 23. Las variables categóricas fueron comparadas estadísticamente mediante el Chi cuadrado y el Odds ratio, con intervalos de confianza al $95 \%$, considerándose un valor de significancia de $\mathrm{p}<0.05$. Además, se efectuó la prueba de regresión logística multivariada.

El proyecto del estudio fue aprobado por la Oficina General de Investigación y Transferencia Tecnológica del INS, mediante Resolución Directoral N. ${ }^{\circ}$ 523-2013-DG-OGITTOPE/INS, con fecha 04 de julio de 2013. 
Cuadro 1. Prevalencia de fasciolosis en niños de edad escolar del distrito de Orurillo, mediante diferentes técnicas de diagnóstico.

\begin{tabular}{lcc}
\hline Prueba & $\mathrm{n}$ & $\%$ \\
\hline Inmunoblot IgG & 33 & 11.2 \\
$\begin{array}{l}\text { ELISA IgG } \\
\begin{array}{l}\text { Sedimentación } \\
\text { rápida }\end{array}\end{array}$ & 68 & 23.1 \\
\hline Total & 5 & 1.7 \\
\hline Valor $\mathrm{p}: 0.000$ & 285 & 100.0 \\
\hline
\end{tabular}

\section{Resultados}

El Cuadro 1 muestra las prevalencia de fasciolosis determinadas mediante los tres métodos empleados en el estudio, observándose que la prueba inmunológica de ELISA
IgG presentó el mayor número de positivos $(23.1 \%$; IC95\%=18.1-28.0) y la prueba coprológica de sedimentación rápida detectó el menor número de muestras positivas $\left(2.3 \% ; \mathrm{IC}_{95 \%}=0.9-7.8\right)$. El Cuadro 2 muestra que mediante el diagnóstico con IB, los factores edad, sexo, nivel educativo y las actividades realizadas por el niño como apoyo en el hogar no mostraron asociación estadística significativa con fasciolosis ( $\mathrm{p}>0.05)$.

La Figura 1 muestra los niveles de infección en los niños según la institución educativa, encontrando que seis de los 12 centros escolares presentaron niveles menores de $15 \%$ de infección, en tanto que los otros seis centros presentaron niveles de infección entre 30 y $50 \%$. Las demás instituciones educativas $(n=11)$ no presentaron casos de fasciolosis, por lo que no son mostradas en la figura. Por otro lado, la Figura 2 muestra que la mayoría de los niños con fasciolosis procedieron de la comunidad de Quishuarani con $66.2 \%$ (2/33); Cachuyo con $33.3 \%$ (1/33) y Choquesani con $21.2 \%(7 / 33)(\mathrm{p}<0.05)$.

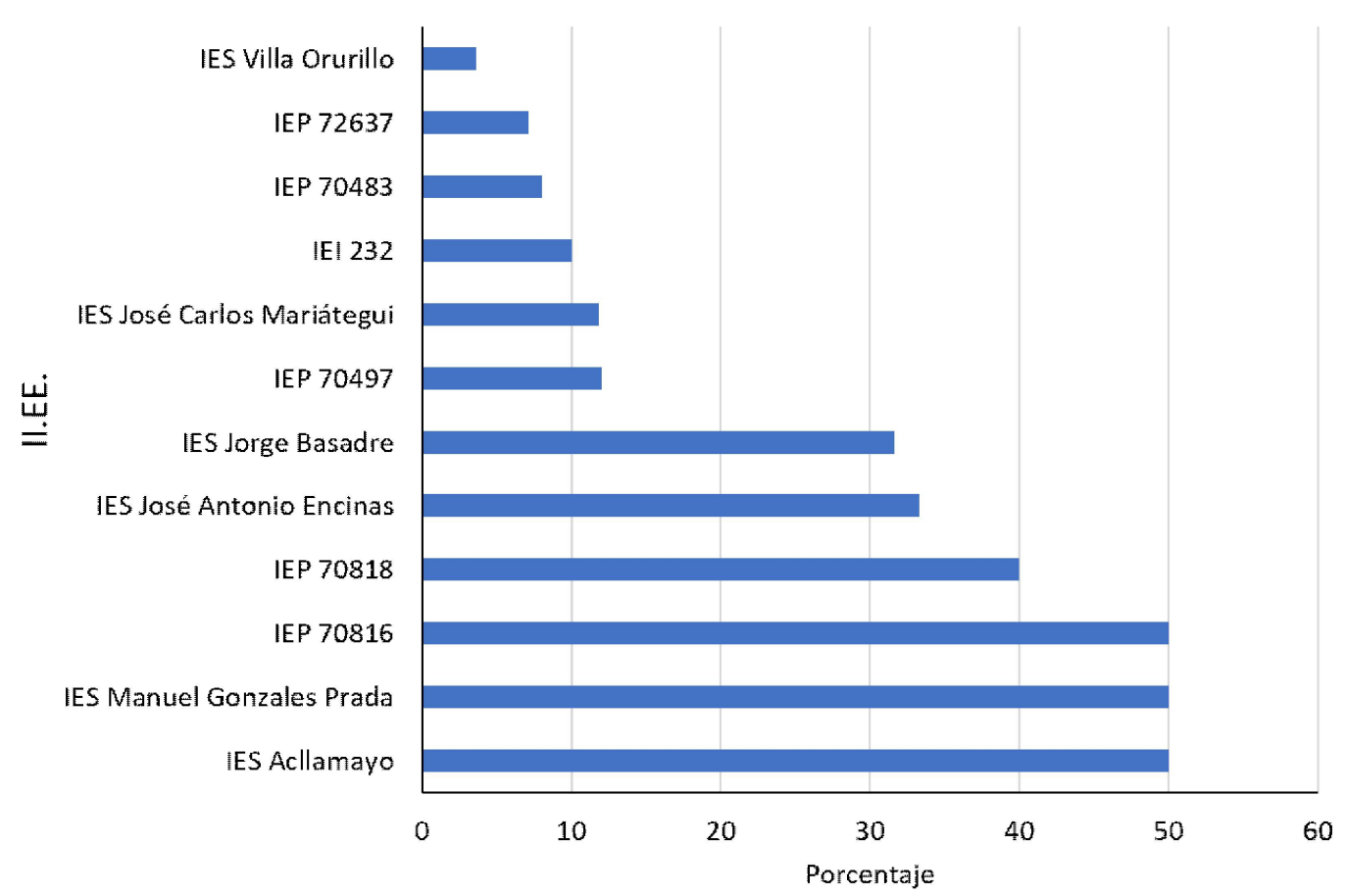

Figura 1. Prevalencia de fasciolosis en niños 9 a 13 años de instituciones educativas (II.EE) del distrito de Orurillo, Puno (2014). $X^{2}=150.039 ; p=0.000$ 
Cuadro 2. Prevalencia de fasciolosis en niños de edad escolar del distrito de Orurillo, Puno (2014)

\begin{tabular}{|c|c|c|c|c|}
\hline & \multicolumn{2}{|c|}{ Fasciolosis } & \multirow{2}{*}{$\begin{array}{l}\text { Total } \\
\text { n (\%) }\end{array}$} & \multirow[b]{2}{*}{$\mathrm{p}$} \\
\hline & $\begin{array}{c}\text { Positivos } \\
\text { n (\%) }\end{array}$ & $\begin{array}{c}\text { Negativos } \\
\text { n (\%) }\end{array}$ & & \\
\hline \multicolumn{5}{|l|}{ Edad (años) } \\
\hline$\leq 5$ & $3(13.6)$ & $19(86.4)$ & 22 & 0.701 \\
\hline $6-8$ & $4(6.9)$ & $54(93.1)$ & 58 & \\
\hline $9-11$ & $9(10.0)$ & $81(90.0)$ & 90 & \\
\hline $12-14$ & $10(14.5)$ & $59(85.5)$ & 69 & \\
\hline$\geq 15$ & $7(12.5)$ & $49(97.5)$ & 56 & \\
\hline \multicolumn{5}{|l|}{ Sexo } \\
\hline Mujer & $20(13.1)$ & $133(86.9)$ & 153 & 0.286 \\
\hline Varón & $13(9.2)$ & $129(90.8)$ & 412 & \\
\hline \multicolumn{5}{|l|}{ Nivel educativo } \\
\hline Inicial & $1(4.3)$ & $22(95.7)$ & 23 & 0.077 \\
\hline Primaria & $13(8.4)$ & 141 (91.6) & 154 & \\
\hline Secundaria & $19(16.1)$ & 99 (83.9) & 118 & \\
\hline \multicolumn{5}{|l|}{ Apoyo al hogar } \\
\hline Pastoreo & $30(12.2)$ & $216(87.8)$ & 246 & 0.218 \\
\hline Agricultura & $22(12.2)$ & $158(87.8)$ & 180 & 0.480 \\
\hline Labores de casa & $6(10.3)$ & $52(89.7)$ & 58 & 0.821 \\
\hline Total & $33(11.2)$ & $262(88.8)$ & 295 & \\
\hline
\end{tabular}

El Cuadro 3 muestra que la fuente de suministro de agua estuvo asociada a fasciolosis $(p<0.05)$. Sin embargo, antecedentes de fasciolosis en la familia, haber viajado en los últimos 90 días, conocer a la $F$. hepatica con sus nombres locales, conocer que el ganado tiene fasciolosis, criar animales, lugar de disposición de excretas, lavado de manos o uso de mondadientes rurales (paja o tallos de vegetales) no mostraron asociación estadística significativa con fasciolosis en los niños ( $p>0.05$ ). Por otro lado, el tipo de alimentos consumido no mostró asociación estadística significativa con fasciolosis en los niños (Cuadro 4), en tanto que, al relacionar el tipo de animal criado en los hogares de los niños, solo la crianza de cuyes estuvo asociada a fasciolosis (Cuadro 5).
El examen coprológico demostró que 123 de 129 niños tuvieron algún tipo de enteroparásitos, $\left(95.4 \% \mathrm{IC}_{95 \%}=91.3-99.4\right)$, donde la mayor frecuencia correspondió a Entamoeba coli (87.6\%; $\left.\mathrm{IC}_{95 \%}=81.5-93.7\right)$, seguido de Endolimax nana (39.5\%, Blastocystis hominis $(34.9 \%)$ y Giardia lamblia (25.6\%). Otros parásitos presentaron frecuencias menores de $10 \%$. Por otro lado, la presencia de enteroparásitos no mostró asociación estadística significativa con fasciolosis (Cuadro 6).

Por último, el análisis multivariado determinó que beber agua de pozo $(\mathrm{OR}=6.9$; $\left.\mathrm{IC}_{95 \%}=2.0-23.6 ; \mathrm{p}=0.002\right)$ y criar cuyes $\left(\mathrm{OR}=4.2 ; \mathrm{IC}_{95 \%}=1.8-9.9 ; \mathrm{p}=0.001\right)$ son factores asociados a fasciolosis en los niños de los centros educativos del distrito bajo estudio. 
Cuadro 3. Factores asociados a la ocurrencia de fasciolosis en niños de edad escolar del distrito de Orurillo, Puno (2014)

\begin{tabular}{|c|c|c|c|c|}
\hline & \multicolumn{2}{|c|}{ Fasciolosis $^{1}$} & \multirow{2}{*}{$\begin{array}{l}\text { Total } \\
\mathrm{n}(\%)\end{array}$} & \multirow[b]{2}{*}{$\mathrm{p}$} \\
\hline & $\begin{array}{l}\text { Positivos } \\
\text { n (\%) }\end{array}$ & $\begin{array}{l}\text { Negativos } \\
\mathrm{n}(\%)\end{array}$ & & \\
\hline \multicolumn{5}{|l|}{ Fuente de suministro de agua } \\
\hline Potable & $4(5.8)$ & $65(94.2)$ & 69 & 0.015 \\
\hline Entubada & $10(8.7)$ & $105(91.3)$ & 115 & \\
\hline Piletas comunitarias & $3(15.0)$ & $17(85.0)$ & 20 & \\
\hline Canales de regadío & - & $1(100.0)$ & 1 & \\
\hline Ríos & - & $8(100.0)$ & 8 & \\
\hline Ojo de agua o manantial & - & $13(100.0)$ & 13 & \\
\hline Pozo & $16(23.2)$ & $53(76.8)$ & 69 & \\
\hline $\begin{array}{l}\text { Antecedentes de fasciolosis } \\
\text { en la familia }\end{array}$ & $2(8.3)$ & $22(91.7)$ & 24 & 0.644 \\
\hline $\begin{array}{l}\text { Haber viajado en los últimos } \\
90 \text { días }\end{array}$ & $14(12.4)$ & $99(87.6)$ & 113 & 0.606 \\
\hline $\begin{array}{l}\text { Conocer a la } F \text {. hepatica como } \\
\text { ccallutaca o alicuya }\end{array}$ & $9(10.2)$ & 79 (89.8) & 88 & 0.733 \\
\hline $\begin{array}{l}\text { Conocer que el ganado tiene } \\
\text { fasciolosis }\end{array}$ & $8(9.3)$ & $78(90.7)$ & 86 & 0.510 \\
\hline Criar animales & $32(11.9)$ & $238(88.1)$ & 270 & 0.233 \\
\hline \multicolumn{5}{|l|}{ Disposición de excretas } \\
\hline Letrina & $29(12.7)$ & $199(87.3)$ & 228 & 0.291 \\
\hline Campo abierto & $1(4.3)$ & $22(95.7)$ & 23 & \\
\hline Desagüe & $3(6.8)$ & $41(93.2)$ & 44 & \\
\hline $\begin{array}{l}\text { Lavado de manos antes } \\
\text { alimentarse y después de ir al } \\
\text { baño }\end{array}$ & $26(11.2)$ & $207(88.8)$ & 233 & 0.944 \\
\hline Uso de mondadientes rurales & $18(11.4)$ & $140(88.6)$ & 158 & 0.922 \\
\hline
\end{tabular}

${ }^{1}$ Según la prueba de Inmunoblot IgG (IB)

\section{Discusión}

El hallazgo de huevos de Fasciola hepatica en deposiciones humanas es poco efectivo debido a la intermitencia en la expulsión de huevos, recomendándose repetir el examen coprológico en varias oportunidades (Maco et al., 2002). Las técnicas inmunológicas tienen la ventaja de poder emplearse en todo el curso de la infección, así como en casos de infecciones extrahepáticas (Maco et al., 2002; Loja et al., 2003). Esta situación fue reconfirmada en el presente estudio, donde el mayor número de casos de fasciolosis fue encontrado mediante la técnica de ELISA frente a la de TSR. Por otro lado, la mayor cantidad de casos positivos detectados a través de la técnica de ELISA en comparación con la de IB podría 


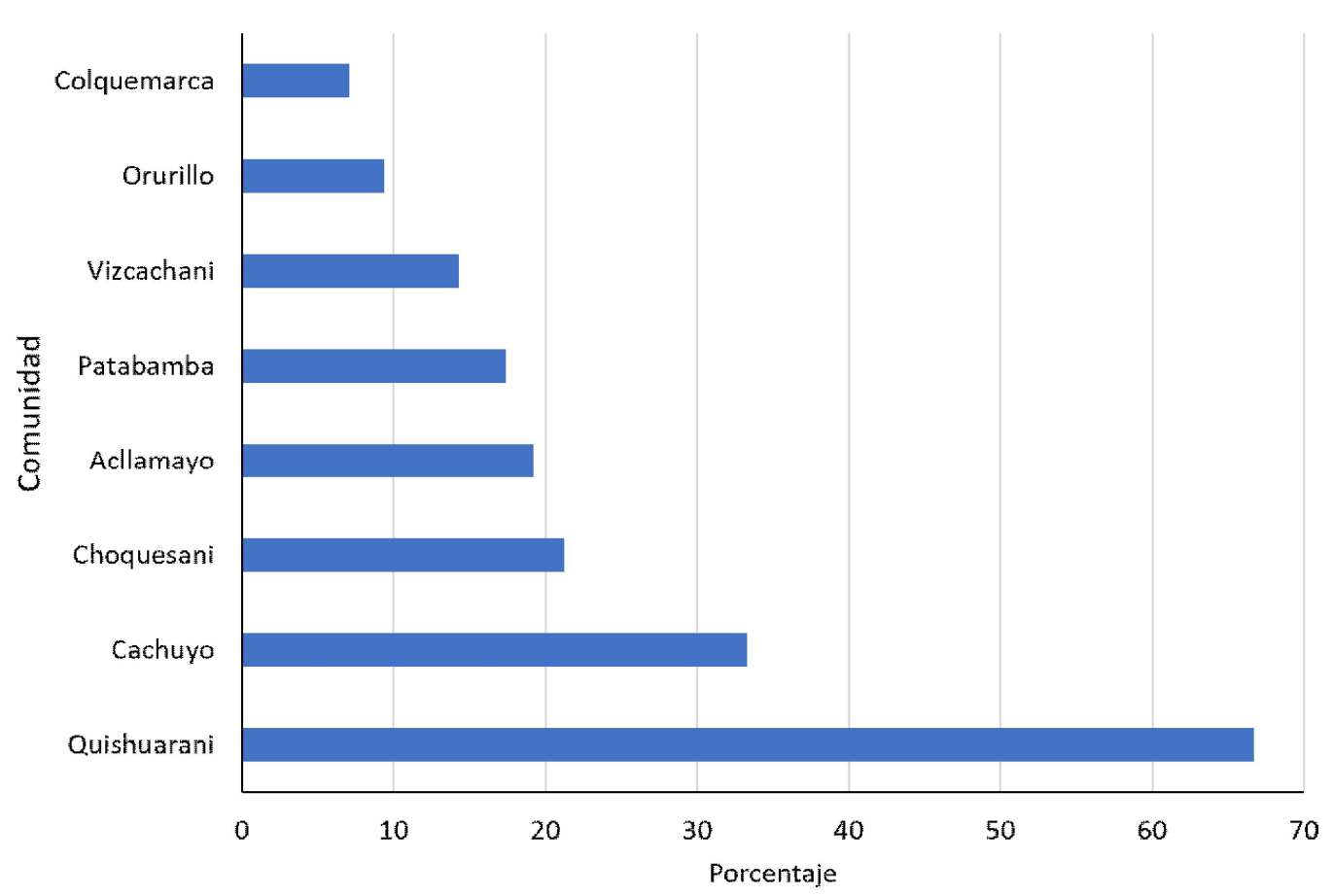

Figura 2. Localidades de procedencia de niños de 9 a 13 años con fasciolosis en comunidades del distrito de Orurillo, Puno (2014). $\mathrm{X}^{2}=109.253$; $\mathrm{p}=0.000$

deberse a la reactividad cruzada con otros helmintos. Esto indica la utilidad de emplear la técnica de ELISA para realizar un tamizaje preliminar de la población y luego la prueba de IB como prueba confirmatoria para obtener al diagnóstico concluyente, superando así la baja sensibilidad del análisis coprológico con TSR (Alarcón de Noya et al., 2007).

La prevalencia de fasciolosis encontrada en este estudio clasifica al distrito de Orurillo como hiperendémico, debido a que supera el 10\% (Mas-Coma et al., 1999; MasComa, 2005), al igual que otras zonas de Perú (Natividad y Terashima, 2008; Ayala et al., 2008; Lopez et al., 2012; León y Cabanillas, 2014). Las zonas endémicas se hallan en regiones altoandinas facilitadas por la presencia de caracoles del género Lymnaea infectados, donde la fase de expulsión de cercarias es más prolongada y el número de metacercarias originadas por el caracol es supe- rior (Mas-Coma et al., 2001; Mas-Coma, 2005). Por otra parte, la prevalencia con fasciolosis encontrada en esta investigación es parecida a lo hallado en zonas hiperendémicas de Puerto Rico (Mas-Coma, 2005) y México (Zumaquero et al., 2013).

La investigación no mostró diferencia estadística significativa en la infección con fasciolosis entre varones y mujeres, probablemente porque las labores de campo y académicas en los centros educativos son compartidas igualitariamente y los hábitos alimenticios son similares. Tampoco se encontró diferencia de fasciolosis entre instituciones educativas de inicial, primaria o secundaria, de manera similar a otras investigaciones (Hassan et al., 1995; Marcos et al., 2005; Valderrama et al., 2019, 2021), no precisamente por ser más proclive al deteriorado sistema sanitario en áreas endémicas, sino a la insuficiente inmunidad existente en este gru- 
Cuadro 4. Relación entre el consumo de alimentos y la seropositivad a fasciolosis en niños de edad escolar del distrito de Orurillo, Puno (2014)

\begin{tabular}{|c|c|c|c|c|}
\hline & \multicolumn{2}{|c|}{ Fasciolosis $^{1}$} & \multirow{2}{*}{$\begin{array}{l}\text { Total } \\
\text { n (\%) }\end{array}$} & \multirow[b]{2}{*}{$\mathrm{p}$} \\
\hline & $\begin{array}{c}\text { Positivos } \\
\mathrm{n}(\%)\end{array}$ & $\begin{array}{c}\text { Negativos } \\
\mathrm{n}(\%)\end{array}$ & & \\
\hline Ensaladas & $32(12.0)$ & $235(88.0)$ & 267 & 0.179 \\
\hline Verduras crudas & $31(11.6)$ & $236(88.4)$ & 267 & 0.476 \\
\hline Berros & $9(11.0)$ & $73(89.0)$ & 82 & 0.943 \\
\hline Lechuga & $30(10.9)$ & 244 (89.1) & 274 & 0.640 \\
\hline Alfalfa & $10(8.5)$ & $108(91.5)$ & 118 & 0.228 \\
\hline Diente de León & $23(13.9)$ & $143(86.1)$ & 166 & 0.104 \\
\hline Agua cruda & $26(12.3)$ & 185 (87.7) & 211 & 0.327 \\
\hline Jugos & $16(10.1)$ & 142 (89.9) & 158 & 0.535 \\
\hline Extractos & $2(28.6)$ & $5(71.4)$ & 7 & 0.140 \\
\hline Emolientes & $2(20.0)$ & $8(80.0)$ & 10 & 0.368 \\
\hline
\end{tabular}

${ }^{1}$ Según la prueba de Inmunoblot IgG (IB)

Cuadro 5. Relación entre la especie de animal de contacto y la seropositividad a fasciolosis en niños de edad escolar del distrito de Orurillo, Puno (2014)

\begin{tabular}{lcccc}
\hline & \multicolumn{2}{c}{ Fasciolosis $^{1}$} & & Total \\
\cline { 2 - 3 } & $\begin{array}{c}\text { Positivos } \\
\mathrm{n}(\%)\end{array}$ & $\begin{array}{c}\text { Negativos } \\
\mathrm{n}(\%)\end{array}$ & & $\mathrm{p}(\%)$ \\
\hline Cuyes & $12(23.5)$ & $39(76.5)$ & 51 & 0.002 \\
Bovinos & $31(12.4)$ & $219(87.6)$ & 250 & 0.119 \\
Ovinos & $29(12.7)$ & $229(87.3)$ & 200 & 0.134 \\
Camélidos & $5(10.4)$ & $43(89.6)$ & 48 & 0.853 \\
Porcinos & $8(8.2)$ & $89(91.8)$ & 97 & 0.262 \\
Equinos & $1(5.9)$ & $16(94.1)$ & 17 & 0.475 \\
Gallinas & $9(15.8)$ & $48(84.2)$ & 57 & 0.220 \\
Perros & $23(13.5)$ & $147(86.5)$ & 170 & 0.137 \\
Gatos & - & $22(100.0)$ & 22 & 0.084 \\
\hline
\end{tabular}

${ }^{1}$ Según la prueba de Inmunoblot IgG (IB) 
Cuadro 6. Relación entre el tipo de enteroparásitos y la seropositividad a fasciolosis en niños de edad escolar del distrito de Orurillo, Puno (2014)

\begin{tabular}{|c|c|c|c|c|}
\hline & \multicolumn{2}{|c|}{ Fasciolosis $^{1}$} & \multirow{2}{*}{$\begin{array}{l}\text { Total } \\
\mathrm{n}(\%)\end{array}$} & \multirow[b]{2}{*}{$\mathrm{p}$} \\
\hline & $\begin{array}{c}\text { Positivos } \\
\mathrm{n}(\%)\end{array}$ & $\begin{array}{c}\text { Negativos } \\
\text { n (\%) }\end{array}$ & & \\
\hline Ascaris lumbricoides $^{\mathrm{h}}$ & - & $6(100.0)$ & 6 & 0.328 \\
\hline Balantidium colit ${ }^{\mathrm{t}}$ & - & $1(100.0)$ & 1 & 0.696 \\
\hline Blastocystis hominis $^{\mathrm{t}}$ & $5(11.1)$ & $40(88.9)$ & 45 & 0.611 \\
\hline Chilomastix mesnili ${ }^{\mathrm{q}}$ & - & $13(100.0)$ & 13 & 0.139 \\
\hline Dientamoeba fragilis ${ }^{\mathrm{t}}$ & - & $2(100.0)$ & 2 & 0.579 \\
\hline Endolimax nana ${ }^{\mathrm{q}}$ & $6(11.8)$ & $45(88.2)$ & 51 & 0.701 \\
\hline Entamoeba coli ${ }^{\mathrm{q}}$ & $14(12.4)$ & $99(87.6)$ & 113 & 0.481 \\
\hline Entamoeba histolytica ${ }^{\mathrm{t}}$ & - & $5(100.0)$ & 5 & 0.374 \\
\hline Enterobius vermicularis ${ }^{1}$ & - & $2(100.0)$ & 2 & 0.579 \\
\hline Giardia lamblia ${ }^{\mathrm{q}}$ & $4(12.1)$ & $29(87.9)$ & 33 & 0.835 \\
\hline Hymenolepis nana ${ }^{\mathrm{h}}$ & - & $6(100.0)$ & 6 & 0.328 \\
\hline Meloidogyne $\mathrm{sp}^{\mathrm{h}}$ & $1(50.0)$ & $1(50.0)$ & 2 & 0.121 \\
\hline Strongyloides stercoralis 1 & - & $1(100.0)$ & 1 & 0.696 \\
\hline Trichomonas hominis ${ }^{\mathrm{t}}$ & - & $6(100.0)$ & 6 & 0.328 \\
\hline
\end{tabular}

${ }^{1}$ Según la prueba de Inmunoblot IgG (IB)

${ }^{\mathrm{h}}$ huevo; ${ }^{\mathrm{t}}$ trofozoíto;' larva; ${ }^{\mathrm{q}}$ quiste

po etario para evadir la infección (Ibáñez et al., 2004). No obstante, otros estudios encontraron diferencias etarias (Albán et al., 2002; Díaz et al., 2011).

Las infecciones disímiles de fasciolosis entre instituciones educativas podría deberse a que las instituciones más apartadas de los centros poblados presentan menos accesibilidad a servicios básicos tales como agua y alumbrado eléctrico (Natividad y Terashima, 2008). Así mismo, la cercanía a acequias aumentaría la posibilidad de infección, ya que los niños utilizan esta agua, en la que se ha verificado que la metacercaria es viable (Marcos et al., 2005). Concomitantemente, los niños que evacúan en letrinas tendrían mejores hábitos de higiene que aquellos que defecan a campo abierto, acequia o río, donde está la fuente de infección (Zumaquero et al., 2013).
También, junto con el agua pueden contaminarse las plantas que, al servir de alimento a los niños, podrían infectarlos (Martínez et al., 2006).

La comunidad de Quishuarani presentó mayor prevalencia de fasciolosis $(66.2 \%)$ debido probablemente a que se encuentra a orillas de un río, a más de $3900 \mathrm{~m}$, alejada de las zonas urbanas y con difícil acceso por carretera, lo que incrementaría el riesgo de infección humana (Valderrama et al., 2021).

La prevalencia con enteroparásitos fue elevada (95.4\%), solo comparable a reportes en localidades peruanas en asentamientos humanos de Ayacucho (91.5\%) (Otárola et al., 2011), y localidades rurales de Loreto (97\%) (Tarqui et al., 2013) y del valle del Mantaro (100\%) (Marcos et al., 2002). La 
elevada frecuencia de enteroparásitos hallada en esta investigación podría deberse al inadecuado saneamiento ambiental e higiene presentes en el ámbito rural, a aspectos socioeconómicos y culturales de la población, a la tupida cobertura vegetal del medio y a la humedad de los suelos areno-arcillosos (Manrique y Suescún, 2011). También, es importante considerar el material del piso de los domicilios, debido a que muchos de ellos son de tierra, lo que podría contribuir a la aparición de estos agentes (Lopez et al., 2012). Además, debe de valorarse los inconsistentes hábitos de higiene y estado nutricional que influyen en un mayor riesgo de infección por protozoos y helmintos en áreas marginales. De otra parte, la investigación demostró que la presencia de enteroparásitos no estuvo asociada con fasciolosis.

Se encontró que Blastocystis hominis, Entamoeba coli, Giardia lamblia y Endolimax nana fueron coinfectantes con Fasciola hepatica, al igual que lo reportado por otros autores (Marcos et al., 2006; Lopez et al., 2012). La manifestación de estos parásitos probablemente se deba a un origen compartido de contagio en los niños, posiblemente mediante el agua; además, a sus incorrectas prácticas de higiene y a la posible contaminación con heces a la que se exponen (Matthys et al., 2011, Valderrama et al., 2021). Cabe mencionar que, el parásito Meloidogyne sp encontrado en este estudio, aunque es un nematodo de las raíces de las plantas, ya había sido reportado excepcionalmente en humanos (Peralta-Siesquen y Rojas-Jaimes, 2020).

El estudio demostró con el análisis multivariado que los niños que beben agua de pozo y crían cuyes tuvieron 6.9 y 4.2 veces más riesgo de fasciolosis, respectivamente. Esto es razonable, ya que beber agua no tratada directa o indirectamente por la contaminación de hortalizas, es considerado como medio de propagación del parásito y al mismo tiempo permite la infección por otros parásitos (Mas-Coma et al., 1999; Lopez et al., 2012; Garaycochea et al., 2012; Zumaquero et al., 2013; Díaz et al., 2014). Además, se debe de considerar que 1173 viviendas en Orurillo se abastecen de agua de pozo (INEI, 2020).

\section{Literatura Cittada}

1. Acha P, Szyfres B. 2003. Zoonosis y enfermedades transmisibles comunes al hombre y los animales. $3^{\mathrm{a}}$ ed. Washington DC: Organización Panamericana de la Salud. 398 p.

2. Alarcón de Noya B, Rojas E, Colmenares $C$, Morales $C$, Contreras $R$, Valero SK, Hernández D, Briceño $S$, et al. 2007. Brote familiar de fascioliasis en Venezuela. Bol Malariol Salud Ambient 47: 47-54.

3. Albán M, Jave J, Quispe T. 2002. Fasciolasis en Cajamarca. Rev Gastroenterol Perú 22: 28-32.

4. Ayala MS, Bustamante ES, González M. 2008. Estado actual de la fascioliasis en Mollebamba, Santiago de Chuco, Región La Libertad y su abordaje por niveles de atención y prevención. Rev Med Vallejiana 5: 89-99.

5. Beltrán M, Tello R, Náquira C. 2003. Manual de procedimientos de laboratorio para el diagnóstico de los parásitos intestinales del hombre. Lima, Perú: Serie de Normas Técnicas INS 37. 90 p.

6. Cornejo H, Oblitas F, Cruzado S, Quispe W. 2010. Evaluación de una prueba de ELISA con antígeno metabólico de Fasciola hepatica para el diagnóstico de fasciolosis humana en Cajamarca, Perú. Rev Peru Med Exp Salud Pública 27: 569-574.

7. Díaz MP, Ramírez NA, Osorio SD. 2014. El sentido de las enfermedades por parásitos intestinales en poblaciones americanas, identificando dilemas bioéticos. Rev Latinoam Bioet 13: 96-111.

8. Díaz R, Garcés M, Millán LM, Pérez J, Millán JC. 2011. Comportamiento clínico-terapéutico de Fasciola hepatica en una serie de 87 pacientes. $\mathrm{Rev} \mathrm{Cu}$ bana Med Trop 63: 268-74. 
9. Escalante H, Davelois K, Ortiz P, Rodríguez H, Díaz E, Jara C. 2011. Estandarización de la técnica de western blot para el diagnóstico de la fasciolosis humana utilizando antígenos de excreciónsecreción de Fasciola hepática. Rev Peru Med Exp Salud Publica 28: 454-461.

10. Espinoza J, Terashima A, Herrera P, Marcos L. 2010. Fasciolosis humana y animal en el Perú: impacto en la economía de las zonas. Rev Perú Med Exp Salud Pública 27: 604-612.

11. Garaycochea $O$, Acosta-García G, Vigo-Ames $N$, Heringman $K$, Dyer A, Jerí S, Siancas G. 2012. Parasitismo intestinal, anemia y estado nutricional en niños de la comunidad de Yantaló, San Martín, Perú. Rev Ibero-Latinoam Parasitol 71: 143-151.

12. Hassan MM, Moustafa NE, Mahmoud LA, Abbaza BE, Hegab MHA. 1995. Prevalence of fasciola infection among school children in Sharkia governorate, Egypt. J Egypt Soc Parasitol 25: 543-549.

13. Ibáñez N, Jara C, Guerra A, Díaz E. 2004. Prevalencia del enteroparasitismo en escolares de comunidades nativas del Alto Marañón, Amazonas, Perú. Rev Peru Med Exp Salud Pública 21_126-133.

14. [INEI] Instituto Nacional de Estadística e Informática. 2020. Lima: Perú en cifras. [Internet]. Disponible en: http:/ /www.inei.gob.pe/

15. Jiménez J, Vergel K, Velásquez M, Vega F, Uscata R, Romero S, Flórez $A$, et al. 2011. Parasitosis en niños en edad escolar: relación con el grado de nutrición y aprendizaje. Rev Horizonte Médico 11: 65-69.

16. León D, Cabanillas O. 2014. Factores de riesgo asociados a fasciolosis humana en tres provincias del departamento de Cajamarca, Perú (periodo 2010). Salud Tecnol Vet 2: 7-13.

17. Loja D, Alvizuri J, Vilca M, Avilés R, Sánchez M. 2003. Hematoma hepático subcapsular por fasciola. Rev Gastroenterol Perú 23: 142-148.
18. Lopez M, Clinton-White A, Cabada MM. 2012. Burden of Fasciola hepatica infection among children from Paucartambo in Cusco, Peru. Am J Trop Med Hyg 86: 481-485.

19. Maco V, Marcos L, Terashima A, Samalvides F, Miranda E, Espinoza J, Gotuzzo E. 2002. Fas2-ELISA y la técnica de sedimentación rápida modificada por lumbreras en el diagnóstico de la infección por Fasciola hepatica. Rev Médica Hered 13: 49-57.

20. Manrique FG, Suescún SH. 2011. Prevalencia de parasitismo intestinal y situación nutricional en escolares y adolescentes de Tunja. Rev CES Med 25: 20-30.

21. Marcos L, Maco V, Samalvidesa F, Terashima A, Espinoza JR, Gotuzzo E. 2006. Risk factors for Fasciola hepatica infection in children: a casecontrol study. T Roy Soc Trop Med H 100: 158-166. doi: 10.1016/j.trstmh.2005.05 .016

22. Marcos L, Maco V, Terashima A, Samalvides F, Espinoza JR, Gotuzzo E. 2005. Fascioliasis in relatives of patients with Fasciola hepatica infection in Peru. Rev Inst Med Trop Sao Paulo 47: 219-222. doi: 10.1590/s0036-46652005000400008

23. Marcos LA, Maco V, Terashima A, Samalvides F, Gotuzzo E. 2002. Prevalencia de parasitosis intestinal en niños del valle del Mantaro, Jauja, Perú. Rev Médica Hered 13: 85-89.

24. Marcos LA. 2007. La infección por Fasciola hepatica en el Perú: una enfermedad emergente. Rev Gastroenterol Perú 27: 389-96.

25. Martínez, I, Gutiérrez M, Romero R, Ruiz L, Gutiérrez EM, Alpizar A, Pimienta RJ. 2006. Seroepidemiology of fascioliasis in school children in Mexico City. Biomédica 17: 251-257.

26. Mas-Coma S, Esteban JG, Bargues MD. 1999. Epidemiology of human fascioliasis: a review and proposed new classification. B World Health Organ 77: 340-346. 
27. Mas-Coma S, Funatsu IR, Bargues MD. 2001. Fasciola hepatica and lymnaeid snails occurring at very high altitude in South America. Parasitology 123: 115-127. doi: 10.1017/S0031182001008034

28. Mas-Coma. 2005. Epidemiology of fascioliasis in human endemic areas. $\mathrm{J}$ Helminthol 79: 207-216. doi: 10.1079/ joh2005296

29. Matthys B, Bobieva M, Karimova G, Mengliboeva $Z$, Jean-Richard $V$, Hoimnazarova M, Kurbonova M, et al. 2011. Prevalence and risk factors of helminths and intestinal protozoa infections among children from primary schools in western Tajikistan. Parasite Vector 4: 195. doi: 10.1186/1756-33054-195

30. Merino K, Valderrama AA. 2017. Fasciola hepatica en bovinos del valle interandino de Aymaraes (Perú): identificación de factores asociados. Rev Med Vet 34: 137-147.

31. [MINEDU] Ministerio de Educación. 2017. Lima: Estadística de la calidad educativa. [Internet]. Disponible en: http://escale.minedu.gob.pe/web/inicio/ padron-de-iiee

32. Natividad IS, Terashima A. 2008. Prevalencia de infección humana por Fasciola hepatica en pobladores del distrito de Caujul provincia de Oyon, región de Lima, Perú. Acta Med Peru 25: 77-80.

33. Otárola J, Beltrán M, Tipacti C, Bellido N. 2011. Prevalencia de enteroparasitosis y factores asociados en niños de 0 a 10 años de edad del asentamiento humano «Juan Velasco Alvarado»-Ayacucho, 2010-2011. En: V Congreso Científico Internacional del Instituto Nacional de Salud. Lima: INS.

34. Peralta-Siesquen C, Rojas-Jaimes J. 2020. Hallazgo de Meloidogyne en una paciente residente de Madre de Dios, Perú. Anal Fac Med 81: 376-377.
35. Sánchez EL, Náquira CG, Vega ES, Miranda EF, Quispe WM, Ayala ER. 2010. Manual de procedimientos para el diagnóstico serológico de las zoonosis parasitarias. Serie de Normas Técnicas No. $32.2^{\circ}$ ed. Lima: Ministerio de Salud. $106 \mathrm{p}$.

36. [SENAMHI] Servicio Nacional de Meteorología e Hidrología del Perú. 2020. Lima: Clima en el Perú. [Internet]. Disponible en: http://www.senamhi.gob.pe/

37. Tarqui K, Beltrán M, Mosqueda R, Otarola J, Solís H. 2013. Enteroparasitosis y factores asociados a su presencia en menores de 15 años procedentes de dos poblaciones del distrito San Juan, Maynas-Loreto 2012. En: VICongreso Científico Internacional del Instituto Nacional de Salud. Lima: INS.

38. Valderrama AA, Serrano DH, Trujillo JM, Merino K, Serrano KJI, Gavidia C, Quispe W. 2019. Crianza de animales domésticos como factor de riesgo de fascioliasis humana. Rev Inv Vet Perú 30: 864-873. doi: 10.15381/rivep.v30i2.16083

39. Valderrama Pomé AA. 2016. Prevalencia de fascioliasis en animales poligástricos de Perú, 1985-2015. Rev Med Vet 32: 121-129. doi: 10.19052/ mv.3861

40. Valderrama AA, Merino K, Serrano KJ, Serrano DH, Trujillo JM, Gavidia CM, Quispe WM, et al. 2021. Asociación de fascioliasis con el estado nutricional y coinfección enteroparasitaria en niños. Rev Cuba Med Tropical 73: 1-18.

41. Zumaquero JL, Sarracent J, Rojas R, Rojas L, Martýnez Y. 2013. Fascioliasis and intestinal parasitoses affecting schoolchildren in Atlixco, Puebla State, Mexico: epidemiology and treatment with nitazoxanide. Plos Neglect Trop D 7: e2553. doi: 10.1371/journal.pntd.0002553 